\title{
Time-series clustering of cytokine expression after transarterial chemoembolization in patients with hepatocellular carcinoma
}

\author{
FENG QI $^{1^{*}}$, LINGXIAO WANG $^{2 *}$, PEIXIN HUANG $^{1 *}$, ZHIYING ZHAO $^{1}$, BIWEI YANG $^{1}$ and JINGLIN XIA $^{1}$ \\ ${ }^{1}$ Liver Cancer Institute, Zhongshan Hospital, Fudan University, Shanghai 200032; ${ }^{2}$ Department of Pediatric Endocrinology \\ and Inherited Metabolic Diseases, Children's Hospital of Fudan University, Shanghai 201102, P.R. China
}

Received Jun 27, 2019; Accepted November 1, 2019

DOI: $10.3892 /$ ol.2019.11209

\begin{abstract}
Cytokines play an important role in the development of tumors. The purpose of the present study was to evaluate the mechanisms and cytokine level changes after transarterial chemoembolization (TACE) in patients with hepatocellular carcinoma (HCC). The Short Time-series Expression Miner (STEM) program was utilized to cluster cytokine expression profiles from the day before TACE to day 21 post-TACE. Based on the identified significant signatures, Gene Ontology (GO) and Kyoto Encyclopedia of Genes and Genomes pathway analyses were performed. Cytokines were serially monitored in 60 evaluable patients to identify the results of the STEM program. Examination of the significant signatures identified 6 significant time-varied expression patterns for 507 cytokines (profiles 16, 18, 28, 41, 42 and 43). GO analysis was enriched in 'cytokine receptor-binding' and 'cytokine receptor activity', and the identified signaling pathways included 'cytokine-cytokine receptor interaction' and the 'JAK-STAT signaling pathway'. Ciliary neurotrophic factor (CNTF) level was increased early after TACE, reaching a peak on day 7 before finally decreasing from day 14 onwards, and was significantly positively correlated with aminotransferase level. Serum levels of pre-TACE IL-10 predicted the local tumor response and overall survival (OS) of the patients, while serum levels of post-TACE IL- $1 \beta$ only indicated the local tumor response of the patient. Overall, the present study identified cytokine time-series expression profiles of patients with HCC undergoing TACE. Early phase increases in CNTF
\end{abstract}

Correspondence to: Professor Jinglin Xia or Professor Bewei Yang, Liver Cancer Institute, Zhongshan Hospital, Fudan University, 180 Fenglin Road, Shanghai 200032, P.R. China

E-mail: xiajinglin@fudan.edu.cn

E-mail: yang.biwei@zs-hospital.sh.cn

*Contributed equally

Abbreviations: HCC, hepatocellular carcinoma; STEM, Short Time-series Expression Miner; TACE, transarterial chemoembolization

Key words: cytokines, HCC, STEM, TACE, prognosis after TACE were associated with post-treatment hepatic injury. IL-1 $\beta$ may reflect an objective response after TACE, while IL-10 may represent a biomarker for OS and the objective response pre-TACE, which may help patients with HCC to benefit from TACE.

\section{Introduction}

Hepatocellular carcinoma ( $\mathrm{HCC}$ ) is a heterogeneous malignant tumor that ranks as the fifth most common primary malignancy and the third leading cause of cancer-associated mortality worldwide (1). Several studies, including studies in North America, Europe, Asia and Australia, have shown that the prognosis is poor in patients with HCC (2-4). Despite recent advances in treatment methods, such as surgical resection and liver transplantation, curative treatment for advanced tumor stages and patients with metastasis is often not feasible. As a standard therapeutic option for intermediate-stage or non-resectable HCC, transarterial chemoembolization (TACE) has been shown to have benefits (5). According to the Barcelona Clinic Liver Cancer (BCLC) staging system, TACE has been identified as an alternative or combination therapy in patients with early or advanced HCC (6). In patients with BCLC stage A, TACE combined with radiofrequency ablation (RFA) exerts better local tumor control compared with that achieved by RFA alone. For patients with BCLC stage C, sorafenib plus TACE significantly delays tumor progression and provides improved survival (7).

Cytokines are a broad and loose category of small proteins that include chemokines, interferons, interleukins, lymphokines and tumor necrosis factors (TNFs), and are produced and released by different cells in the liver; cytokines play an important role in inflammation and tumor progression (8). As such, TNF- $\alpha$ acts as a master switch in establishing an intricate link between proinflammation and disease progression in patients with glioblastoma (9). Through a granulocyte-macrophage colony-stimulating factor- and interleukin (IL)-5-dependent mechanism, obesity-mediated low-grade inflammation increases breast cancer metastasis to the lung (10). IL-10 is associated with numerous malignancies, including non-small cell lung cancer (NSCLC), diffuse large B-cell lymphoma, and cervical and oral cancer (11-15). Moreover, Zhang et al (16) identified a hypoxia-inducible factor (HIF)- $1 \alpha /$ IL- $1 \beta$ signaling loop in the tumor microenvironment that results in liver cancer 
cell epithelial-mesenchymal transformation (EMT) and metastasis. Chemokine ligand 5 (CCL5) plays an important role in the process of chronic liver inflammation, which leads to HCC development by reducing immune cell infiltration, such as $\mathrm{CD} 4^{+}$and $\mathrm{CD} 8^{+} \mathrm{T}$ cells (17).

To date, little is known about the detailed changes that occur in cytokine profiles after TACE. Due to its rarity, the majority of previous studies have focused on individual cytokines, and prior to the present study, few studies have explored changes in serum cytokines, signaling pathways and disease prognosis based on microarray data (18-22). The general focus of the present study was to identify differences in the cytokine expression profiles of patients with HCC undergoing TACE with a specific interest in identifying significantly changed biological processes and Kyoto Encyclopedia of Genes and Genomes (KEGG) pathways. The specific aims of the present study were to investigate the associations among time-dependent changes in cytokine panels and immune reactions, and to evaluate the circulating levels of cytokines as prognostic markers.

\section{Materials and methods}

Patients. A total of 60 patients admitted to the Zhongshan Hospital (Shanghai, China) who received TACE therapy at the Liver Cancer Institute, and 10 healthy controls ( $\geq 18$ years old; 5 males and 5 females), were prospectively recruited between January 2013 and December 2014 and enrolled in the present study (the patient characteristics are shown in Table I). All patients possessed a diagnosis of $\mathrm{HCC}$ according to histological evidence or elevated $\alpha$-fetoprotein (AFP) levels $(>200 \mathrm{ng} / \mathrm{ml}$ ) with positive radiological findings using $\mathrm{CT} / \mathrm{MRI}$. The primary inclusion criteria were as follows: i) Hepatitis B virus as the cause of HCC; ii) patients with Child-Pugh class A or B who had a non-resectable tumor (23); iii) an age $\geq 18$ years; iv) definite BCLC staging (6); and v) clinical and laboratory data, including biochemical parameters, hematological parameters, such as albumin, AFP, alanine transaminase and bilirubin, and tumor sizes, which were recorded at baseline and post-TACE. All chemoembolization procedures were conducted via the right femoral artery and all patients undergoing treatment via TACE were examined by computed tomography or magnetic resonance imaging to evaluate the curative effect based on RECIST 1.1 and mRECIST (24), designated as complete response (CR), partial response (PR), stable disease (SD), or progressive disease (PD). CR and PR were further summarized into objective response (OR). Patients were excluded if there was no clear survival time. A total of 10 patients $(\geq 18$ years old; 5 males and 5 females) were randomly selected from the 60 patients with HCC to compare their serum cytokine levels with those of healthy controls, with the samples collected at the pre-intervention time point. Informed written consent was provided by each patient, and the present study was approved by the Ethics Committees of Zhongshan Hospital. The study protocol was conducted in accordance with the ethical standards specified in the Declaration of Helsinki.

Treatment and follow-up laboratory data. Clinical and laboratory data of enrolled patients were recorded at baseline (pre-TACE day 0) and at regular intervals (post-TACE day 3,7 ,
14 and 21) during the follow-up period, including patient age, sex, hematologic parameters, alanine/aspartate aminotransferases (AST/ALT), albumin, total bilirubin and portal vein invasion. The 60 patients with $\mathrm{HCC}$ were subsequently divided into 2 groups based on tumor size $(\leq 5$ and $>5 \mathrm{~cm})$ and ALT levels ( $\leq 40$ and $>40 \mathrm{U} / \mathrm{l})$ for further analysis.

Microarray data and processing. Serum samples were collected prior to TACE therapy and again on days 3,7, 14 and 21 after the procedure. All samples were stored at $-80^{\circ} \mathrm{C}$, and 9 serum samples randomly selected from the 60 patients with HCC were labelled (Table SI), hybridized and washed using an Agilent hybridization 5188-5242 kit (Agilent Technologies, Inc.). Slides were analysed using an Agilent Microarray Scanner with Feature Extraction G4463AA software (Agilent Technologies, Inc.). Each serum sample obtained pre-TACE (day 0) was compared with those obtained on days 3, 7, 14 and 21 and used the limma package of R 3.6.1 software (25) to identify differentially expressed cytokine genes (DECs). $\mathrm{P}<0.05$ and $\mid \log$ fold-change (FC) $\mid>1$ were counted as detected signals. Subsequently, the pheatmap package in R 3.6.1 software (25) was used to construct a heat map of differentially expressed genes. To ensure specificity, the DECs of all four groups (day $3,7,14$ and 21 vs. day 0 , respectively) were integrated using venn2.1 (http://bioinfogp.cnb.csic.es/tools/venny/index.html).

Short time-series expression miner (STEM). First, the STEM algorithm and 1.3.12 software was used to analyse the dataset using the 507 identified cytokine microarray (http://www. cs.cmu.edu/ jernst/stem) (26). Following the input phase, the STEM clustering algorithm was executed and displayed the clustering results. The clustering algorithm that STEM implements takes advantage of a point-in-time of the dataset and selects a set of distinct and representative temporal expression profiles called model profiles (Fig. 1A). Each gene was assigned to the model profile that most closely matched that gene's expression profile as determined by the correlation coefficient. Next, the standard hypothesis testing was used to determine which model profiles had significantly more genes assigned to them under the true ordering of the time points in the permutation runs. The parameters used for STEM clustering were set at a maximum of 50 model profiles, a maximum unit change between time points of 2 and a minimum correlation for clustering similar profiles $>0.7$. Finally, clusters based on the expression trend of significant genes were screened out $(\mathrm{P}<0.05)$.

Gene Ontology (GO) and signaling pathways. DECs were determined by means of a significance analysis of the microarray and model profile data. $\mathrm{GO}$ analysis determined that there were 284 DECs through use of the Database for Annotation, Visualization and Integrated Discovery (https://david.ncifcrf. gov/) $(27)$ online tool $(\mathrm{P}<0.05)$. The signaling pathways that were likely impacted by the DECs were predicted using the KEGG pathway map tool $(\mathrm{P}<0.05)(28)$.

Protein-protein interaction (PPI) network. The Search Tool for the Retrieval of Interacting Genes/Proteins (STRING) 5.18 (https://string-db.org/) is a repository of known and predicted protein interrelationships (29) and was used to identify possible interacting proteins between the DECs. 
Table I. Characteristics of 60 patients with hepatocellular carcinoma treated with transarterial chemoembolization.

\begin{tabular}{lc}
\hline Characteristic & No. of patients $(\%)$ \\
\hline Age, years & \\
$<50$ & $17(28.3)$ \\
$\geq 50$ & $43(71.7)$ \\
Sex & \\
Female & $8(13.3)$ \\
Male & $52(86.7)$ \\
Albumin, g/l & \\
$\geq 35$ & $53(88.3)$ \\
$<35$ & $7(11.7)$ \\
AFP, ng/ml & \\
$\leq 20$ & $16(26.7)$ \\
$>20$ & $44(73.3)$ \\
Tumor diameter, cm & \\
$\leq 5$ & $22(36.7)$ \\
$>5$ & $38(63.3)$ \\
Portal vein invasion & \\
Without & $39(65.0)$ \\
With & $21(35.0)$ \\
BCLC stage & \\
A & \\
B/C & $55(8.3)$ \\
Serum IL-10 pre-TACE, pg/ml & \\
$<1.59$ & $30(50.0)$ \\
$>1.59$ & \\
\hline
\end{tabular}

AFP, $\alpha$-fetoprotein; BCLC, Barcelona Clinic Liver Cancer; IL, interleukin.

ELISA. Serum samples were collected pre-TACE and on days $3,7,14$, and 21 after the initiation of treatment from 60 evaluable patients who underwent transarterial chemotherapy. The cytokine levels of IL-1B, IL-10, IL-5 and CNTF were analysed using a multiplex immunoassay Bio-Plex 200 array system and Bio-Plex Manager v6.0 software [Hangzhou Multisciences (Lianke) Biotech Co., Ltd] according to the manufacturer's instructions.

Statistical analysis. Student's t-test was used for continuous data, and $\chi^{2}$ test was used for categorical data. The comparison of multiple groups was performed with ANOVA and Tukey's post hoc test. The criteria for screening DECs were $\mid \log \mathrm{FCl}>1.0$ and a false discovery rate $<0.05$. Spearman's correlation was also used to measure the correlation between different cytokine levels as well as between cytokine levels and clinical data. Receiver operating characteristic (ROC) curves were used by plotting sensitivity against 1-specificity. The optimal cut-off values for ROC curves were obtained using the Youden index. The survival curve was plotted using the Kaplan-Meier method, and the overall survival (OS) rate between subgroups was compared using the log-rank test. The Cox proportional hazard regression model was used to evaluate prognostic factors, and hazard ratio (HR) and $95 \%$ confidence interval was determined. The aforementioned statistical data were analysed using SPSS v22.0 software (IBM, Corp.) and GraphPad Prism 6 (GraphPad Software, Inc.). $\mathrm{P}<0.05$ was considered to indicate a statistically significant difference.

\section{Results}

Identification of time-series cytokine genes after TACE. The Agilent cytokine genome microarrays were used to measure the relative gene expression levels in 9 patients with HCC pre-TACE and on days 3, 7, 14 and 21 post-TACE. In addition, 9 patients with $\mathrm{HCC}$ were selected for microarray analysis based on objective response, including three OR and six non-OR patients. A total of 507 cytokine genes were identified and the differentially expressed genes on days 3, 7, 14 and 21 were compared with day 0 . Given the limited overlap of the differentially expressed genes among the four datasets (data not shown), only five genes, including neural differentiation 1 (NEUROD1), triggering receptor expressed on myeloid cells 1 (TREM1), neuregulin 3 (NRG3), Charcot-Leyden crystal galectin (CLC) and TNF $\alpha$-induced protein 6 (TNFAIP6) were identified. Hence, the relevant gene clusters were identified based on the STEM algorithm. The input parameters $\mathrm{c}=2$ and $\mathrm{m}=50$ were used, where $\mathrm{c}$ indicates the units of change and $\mathrm{m}$ indicates the number of candidate profiles. In all, 284 out of 507 differentially expressed genes were significantly clustered in eight models, including profiles $6,14,16,18,28,41,42$ and 43. However, $<10$ genes were clustered in profiles 6 and 14 and therefore were eliminated in further analysis (Fig. 1A). Fig. 1 shows the fold-changes and P-values of the gene expression profiles for the six significant clusters, and their gene symbols and relative expressions are listed in Table SII. Based on STEM analysis, CTNF and IL-5 were found to cluster in profile 18, IL-1 $\beta$ and IL-10 were found to cluster in profile 41, CCL2 and MMP9 were found to cluster in profile 43, CCR7 and CCR1 were found to cluster in profile 16, INS and BMP7 were found to cluster in profile 42, and IL-4 and IL-17RA were found to cluster in profile 28 (Fig. 1B-E and Table II). The trend curves of the gene signatures clustered in profiles 28 , 41,42 and 41 were similar and were all gradually upregulated (Fig. 1B-G). However, some cytokines were not significant in this study, such as IL-6 and IL-8 associated with TACE prognosis. The trend result of CTNF, IL-5, IL-1 $\beta$ and IL-10 was identified according to ELISA data (Fig. 2A-D). Subsequently, the baseline serum levels of CTNF, IL-5, IL-10 and IL- $1 \beta$ from patients with HCC who underwent TACE and healthy controls were compared. The pre-TACE serum levels of CTNF, IL-5, IL-10 and IL-1 $\beta$ were significantly elevated compared with that in the healthy controls (Fig. 2E-H).

GO and KEGG pathway analyses of time-series cytokine genes after TACE. Based on the six significant clusters, GO analysis was performed. The results demonstrated that the DECs in the six significant clusters were related to 'cytokine receptor binding', 'receptor ligand activity' and 'cytokine activity' (Fig. 3A). In profile 18, the DECs were also enriched in 'growth factor receptor binding'. 


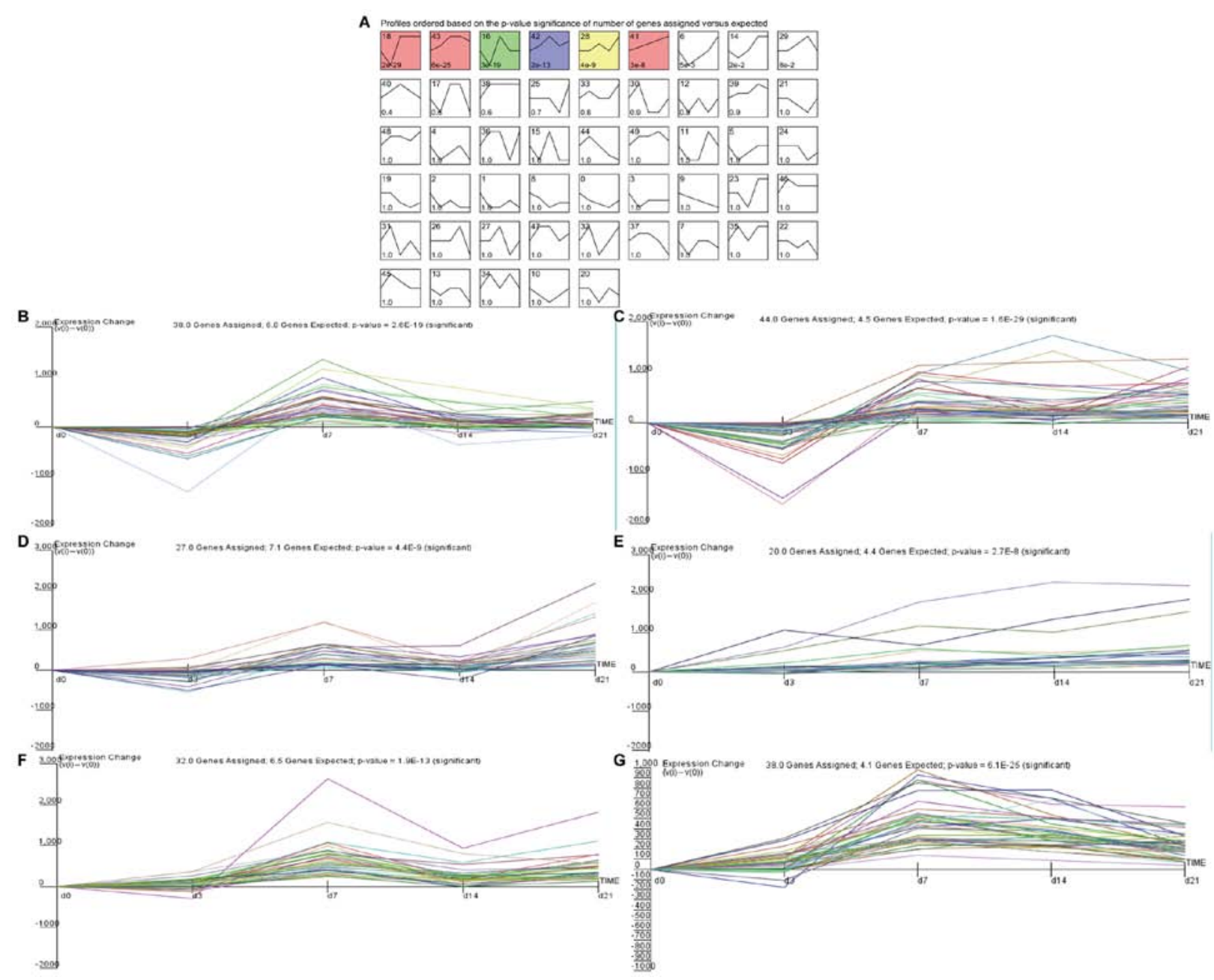

Figure 1. Dynamic expression pattern profiles of differentially expressed cytokines after TACE. (A) Short time-series expression minor clustering analysis was performed to identify clusters. In all, 284 out of 507 of the differentially expressed genes were significantly clustered in six models, including (B) profile 16 , (C) profile 18, (D) profile 28, (E) profile 41, (F) profile 42 and (G) profile 43. The x-axis represents the time point after TACE. The time series was log-normalized and starts at 0 . The $y$-axes of all the genes in a cluster box are shown on the same scale. The different colours in each profile represent different cytokines clustered in each profile. TACE, transarterial chemoembolization.

According to STEM analysis, KEGG signaling pathways were examined to determine whether they were dysregulated in the six significant clusters. 'Cytokine-cytokine receptor interactions' and 'JAK-STAT signaling' were significantly different in all profiles, with the exception of profile 41 (Fig. 3B). The IL-17 signaling pathway was present in four of the six significant clusters (profile 18, 28, 42 and 43) and included IL-5, IL-4, IL-1 $\beta$, IL-17D and CCL2. Intriguingly, the signaling pathway exhibited differences in profile 41 , which included cytokines IL-1 $\beta$ and IL-10. Likewise, the MAPK/RAS and Toll-like receptor signaling pathways, which are associated with cancer (30), were detected in profiles 16 and 43 , and profiles 16 and 41 , respectively, suggesting that these cytokines could be involved in inflammation and cancer transformation in HCC. The CNTF, colony stimulating factor 2 receptor $\alpha$ subunit, interferon- $\gamma$, IL-5, platelet-derived growth factor receptor $\beta$ (PDGFRB), prolactin (PRL) and IL-27 receptor subunit $\alpha$ genes were differentially expressed and enriched in the JAK-STAT signaling pathway in profile 18. Similar to profile 18, the DECs and pathways clustered in profile 16 also included inflammatory signaling pathways (the JAK-STAT and NF-kB pathways), as well as CCR1, CCR7, ICAM1 and BMP4 (Table SIII).
PPI network. To examine the associations among the DECs in each significant cluster, a PPI network was constructed (Fig. 4). The STRING database was used to identify possible interactions of each cytokine expression profile. The results demonstrated that the 32, 39, 21, 18, 28 and 34 cytokines identified in profiles $16,18,28,41,42$ and 43 , respectively, were interacting with each other. In each profile, the high degree of cytokines, which were based on the degree and the association with tumors, including CTNF, IL-5, IL-10 and IL-1 $\beta$, were selected. Additionally, the cytokines with the highest association in each respective cluster were CCR7, TNF, IL-4, IL-10, INS and CCL2 (Table II).

Correlation between liver function and the post-treatment cytokine CNTF levels. The association between the level of ALT or AST and the CNTF cytokine levels during treatment was investigated in patients with $\mathrm{HCC}$ undergoing TACE. There was no significant correlation between serum ALT $(\mathrm{P}=0.53)$ or AST $(\mathrm{P}=0.27)$ levels and CNTF levels on post-treatment day 3. Positive correlations with CNTF levels appeared over time and were significant on day 7 for the ALT $(r=0.68 ; \mathrm{P}<0.01)$ and AST $(r=0.54 ; \mathrm{P}<0.01)$ levels. However, 

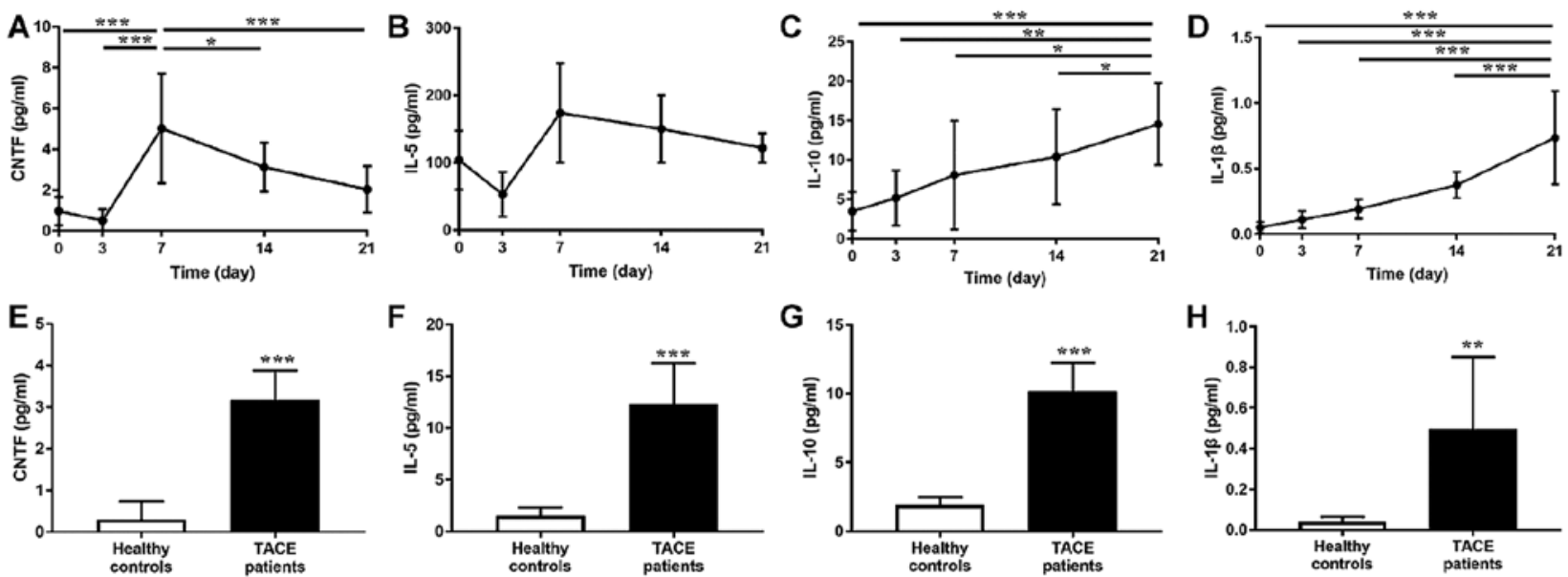

Figure 2. Identification of the expression changes of selected cytokines. The serum levels of (A) CNTF and (B) IL-5 showed a significant early-phase increase after TACE compared to baseline ( $\mathrm{n}=60)$. The serum levels of (C) IL-10 and (D) IL-1 $\beta$ showed a persistent elevation after TACE compared to baseline ( $\mathrm{n}=60$ ). Data was analysed using ANOVA followed by Tukey's post hoc test. The pre-TACE serum levels of (E) CNTF, (F) IL-5, (G) IL-10 and (H) IL-1 $\beta$ were significantly elevated in patients with HCC (healthy controls $n=10$ vs. patients treated with TACE $n=10$ ). Data was analysed using unpaired t-test. Data are expressed as the mean \pm SEM from a minimum of three independent experiments. ${ }^{*} \mathrm{P}<0.05 ;{ }^{* * *} \mathrm{P}<0.01 ;{ }^{* * * *} \mathrm{P}<0.001$. SEM, standard error of the mean; TACE, transarterial chemoembolization; CNTF, ciliary neurotrophic factor; IL, interleukin.
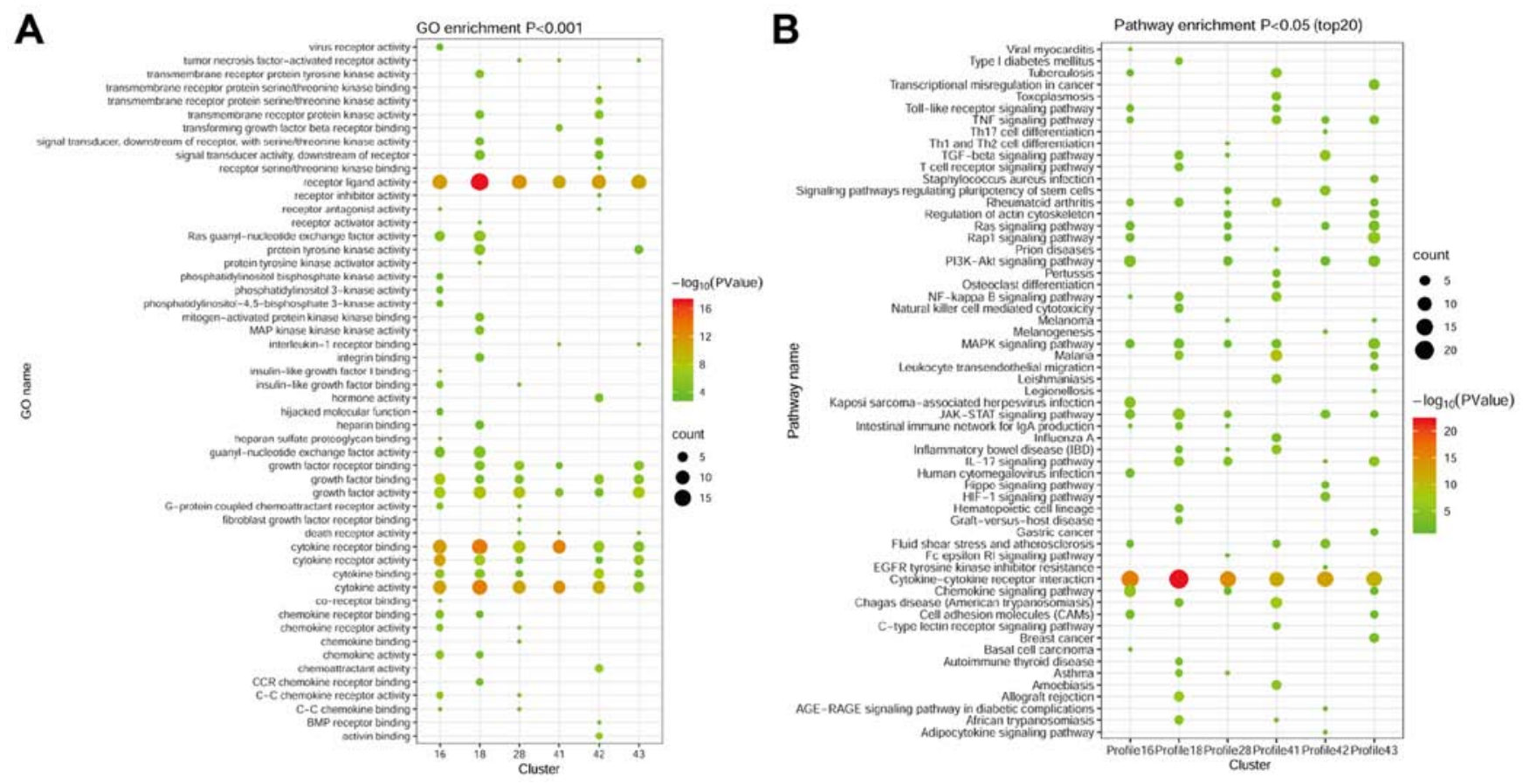

Figure 3. Bioinformatics analysis of differentially expressed genes. (A) GO analysis, according to the cytokines clustered in each the 6 significant cluster profiles, showed $>20$ gene functions. (B) Kyoto Encyclopedia of Genes and Genomes pathway enrichment analysis, according to the cytokines clustered in each the 6 significant cluster profiles, showed $>20$ pathways. GO, Gene Ontology.

on days 14 and 21 after TACE, there was no longer a correlation between the level of ALT or AST and the CNTF levels (Fig. 5A-D). Subsequently the changes in ALT and CNTF levels between small and large HCC tumors were measured. In 60 patients with $\mathrm{HCC}$, those with large tumors $(>5 \mathrm{~cm})$ exhibited more frequent episodes of severe hepatitis (ALT elevation $>40 \mathrm{U} / \mathrm{l}$ ) compared with those with small tumors [36/38 (94.7\%) vs. 15/22 (68.2\%); Fig. 5E and F]. Patients with large tumors $(>5 \mathrm{~cm})$ had a higher baseline and greater increases in CNTF day 7 after TACE, whereas patients with small HCC $(\leq 5 \mathrm{~cm})$ exhibited only minor rises in CNTF levels.
This characteristic change in CNTF that occurred following TACE suggests that there is an increase in CNTF levels in patients with tumors $>5 \mathrm{~cm}$, on day 7 .

Pretreatment serum levels of IL-10 and post-treatment serum levels of $I L-1 \beta$ predict an objective response to TACE therapy. Subsequently it was investigated whether the serum levels of IL-10 and IL-1 $\beta$ had predictive value regarding the trend curve from the microarray data observed for profile 41 (Fig. 1E). The correlations between serum AFP and IL-10/IL-1 $\beta$ were investigated, and there was a significant correlation between 
A

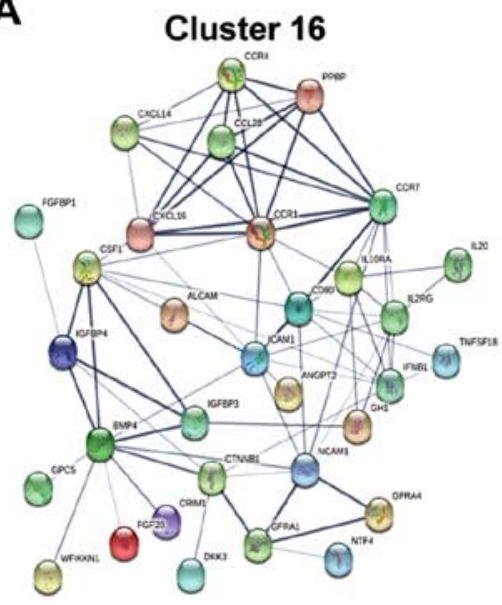

D

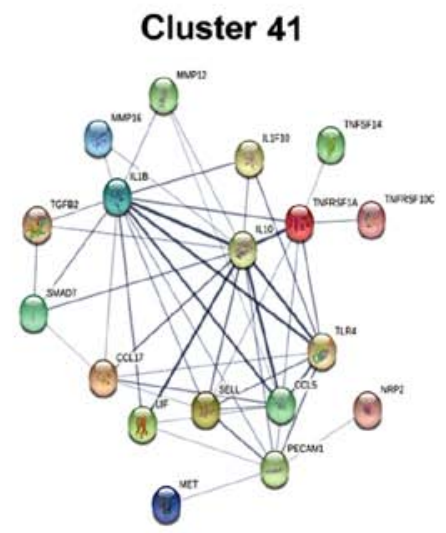

B

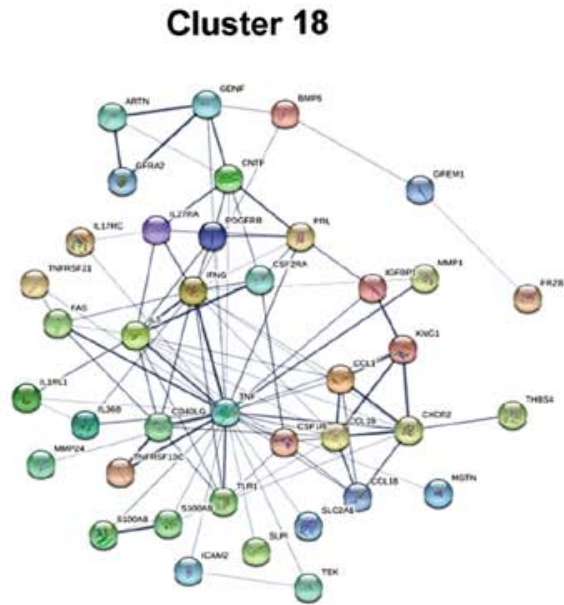

E

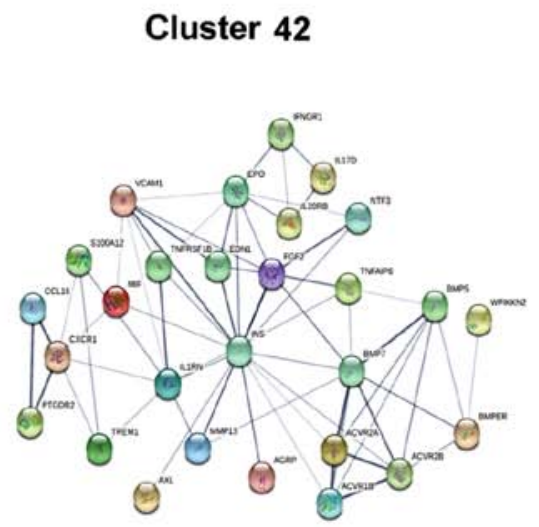

C

Cluster 28

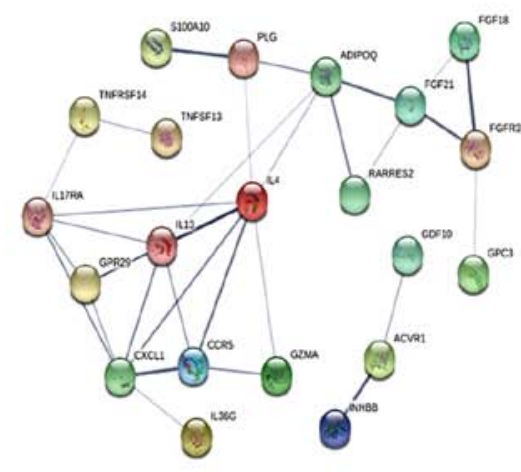

$\mathbf{F}$

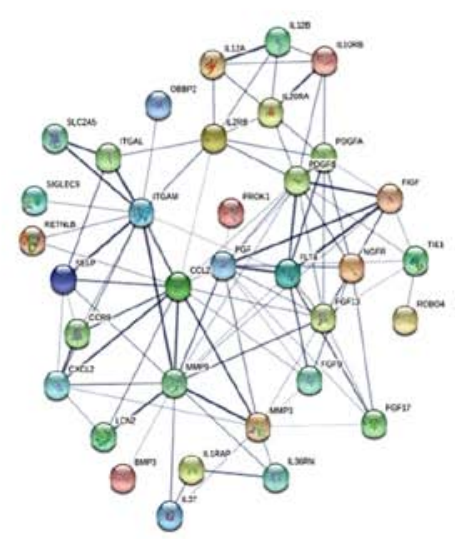

Figure 4. Protein-protein interaction network of each module predicated using Search Tool for the Retrieval of Interacting Genes/Proteins. The network of cytokines clustered in (A) profile 16, (B) profile 18, (C) profile 28, (D) profile 41, (E) profile 42 and (F) profile 43 . The lines represent the correlation of cytokines, and the thickness represents the degree of correlation.

the serum AFP and pre-TACE IL-10 levels (Fig. 6A and B). However, tumor diameter was positively associated with IL-1 $\beta$ levels, and patients with large tumors $(>5 \mathrm{~cm})$ had higher baseline levels and greater increases in post-TACE levels of IL-1 $\beta$ (Fig. 6C and D). This characteristic pattern of IL-1 $\beta$ release might be associated with larger tumors, which have increased hypoxic areas. However, there was not a significant association between the serum levels of IL-10 and IL-1 $\beta$ and BCLC stage, including BCLC stage $\mathrm{A}(\mathrm{n}=5)$ and $\mathrm{B} / \mathrm{C}(\mathrm{n}=55)$ (Fig. $6 \mathrm{E}$ and $\mathrm{F})$ on day 0 pre-treatment and day 3 post-treatment.

The cohort was further divided into two groups: Patients with an OR (complete or partial remission; $n=13$ ) and patients who showed a non-OR (stable or progressive disease; $\mathrm{n}=47$ ). In the analysis, the pretreatment levels of IL-10 and post-treatment levels of IL- $1 \beta$ were significantly higher in patients who showed a non-OR after TACE compared with that in patients with an OR, while the post-treatment levels of IL-10 and pretreatment levels of IL-1 $\beta$ were not significantly different between OR and non-OR patients (Fig. 6G and $\mathrm{H}$ ). In line with this finding, ROC curve analysis of the differences between patients with either an OR or non-OR revealed that the area under the curve (AUC) value of 0.736 for pre-TACE IL-10 was higher than that for post-TACE IL-10 (AUC, 0.508 ) and that the AUC value of 0.726 for post-TACE
IL-1 $\beta$ was higher than that for pre-TACE IL-1 $\beta$ (AUC, 0.661) (Fig. 6I and J).

Serum levels of pretreatment IL-10 predict OS time after TACE. Based on the predictive value of the pretreatment serum levels of IL-10 and post-treatment serum levels of IL-1 $\beta$, the serum levels of IL-10 and IL-1 $\beta$ was subsequently evaluated to determine whether they influenced patient OS. Based on the Youden index of the ROC curve analysis, which is used to identify the ideal cut-off values for cytokines, Kaplan-Meier analysis showed that patients with pretreatment serum levels of IL-10 above the ideal cut-off value $(1.59 \mathrm{pg} / \mathrm{ml})$ experience reduced survival compared to patients with levels below the ideal cut-off values [mOS: 13.55 months vs. undefined (median OS not reached); $\mathrm{P}=0.03$ ] (Fig. 7A). By contrast, the ideal cut-off values for post-treatment IL-10, pretreatment IL-1 $\beta$ and post-treatment IL-1 $\beta$ levels did not effectively identify patients with a poor prognosis (Fig. 7B-D).

To further investigate prognostic factors, the results of the Kaplan-Meier analysis for OS were further analysed using univariate and multivariate Cox regression models. Fig. 7E summarizes the univariate factor analysis, and the HR for IL-10 was 2.193 ( $\mathrm{P}=0.037$; 95\% CI, 1.048-4.589; Table III). Subsequently parameters with $\mathrm{P}<0.70$ from the univariate Cox 
Table II. Degree of gene modulation in the protein-protein interaction network.

\section{A, Genes in profile 18}

\begin{tabular}{lc}
\hline Gene & Degree \\
\hline TNF & 30 \\
IFNG & 15 \\
IL5 & 13 \\
CCL19 & 10 \\
CXCR2 & 10 \\
TLR1 & 9 \\
CD40LG & 7 \\
CCL1 & 7 \\
CNTF & 7 \\
PRL & 7 \\
\hline
\end{tabular}

$\mathrm{B}$, Genes in profile 43

\begin{tabular}{lc}
\hline Gene & Degree \\
\hline CCL2 & 15 \\
MMP9 & 14 \\
PGF & 13 \\
ITGAM & 12 \\
PDGFB & 12 \\
FGF13 & 11 \\
FLT4 & 9 \\
MMP3 & 9 \\
PDGFA & 9 \\
FIGF & 8 \\
\hline
\end{tabular}

$\mathrm{C}$, Genes in profile 41

\begin{tabular}{lc}
\hline Gene & Degree \\
\hline IL10 & 13 \\
IL1B & 13 \\
CCL5 & 9 \\
TLR4 & 8 \\
PECAM1 & 8 \\
TNFRSF1A & 7 \\
SELL & 7 \\
CCL17 & 6 \\
LIF & 6 \\
SMAD7 & 4 \\
\end{tabular}

$\mathrm{D}$, Genes in profile 16

Gene

Degree

\section{CCR7}

ICAM1
Table II. Continued.

\begin{tabular}{|c|c|}
\hline Gene & Degree \\
\hline CD80 & 9 \\
\hline NCAM1 & 8 \\
\hline CXCL16 & 8 \\
\hline IFNB1 & 8 \\
\hline IL2RG & 8 \\
\hline \multicolumn{2}{|c|}{$\mathrm{E}$, Genes in profile 42} \\
\hline Gene & Degree \\
\hline INS & 15 \\
\hline BMP7 & 9 \\
\hline FGF2 & 8 \\
\hline IL1RN & 8 \\
\hline EPO & 8 \\
\hline VCAM1 & 7 \\
\hline ACVR2B & 6 \\
\hline CXCR1 & 6 \\
\hline BMP5 & 6 \\
\hline ACVR1B & 5 \\
\hline \multicolumn{2}{|c|}{ F, Genes in profile 28} \\
\hline Gene & Degree \\
\hline IL4 & 8 \\
\hline IL13 & 6 \\
\hline CXCL1 & 6 \\
\hline ADIPOQ & 5 \\
\hline IL17RA & 5 \\
\hline CCR5 & 4 \\
\hline FGF21 & 4 \\
\hline GPR29 & 4 \\
\hline FGFR3 & 3 \\
\hline PLG & 3 \\
\hline
\end{tabular}

regression models (IL-10, tumor diameter, portal vein invasion, BCLC stage B/C vs. A, albumin and AFP) were removed and multivariate Cox analysis (Table IV) was performed. Importantly, the prognostic value of the pretreatment serum level of IL-10 was independent of those parameters.

\section{Discussion}

TACE is considered one of the most effective treatments for non-resectable liver cancer (31). At present, numerous studies have reported that changes in cytokines occur in patients with liver cancer after TACE, and these changes may be related to acute inflammatory reactions, acute liver injury and prognostic factors $(18,19)$. However, the potential mechanisms involving these cytokines in patients with a post-TACE prognosis have not been completely elucidated. In the present study, the 

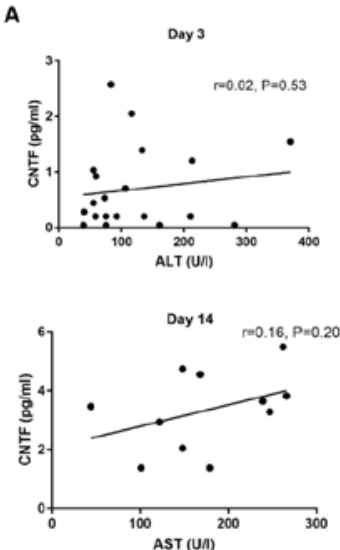
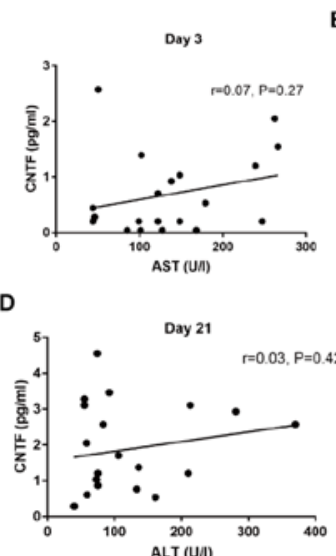
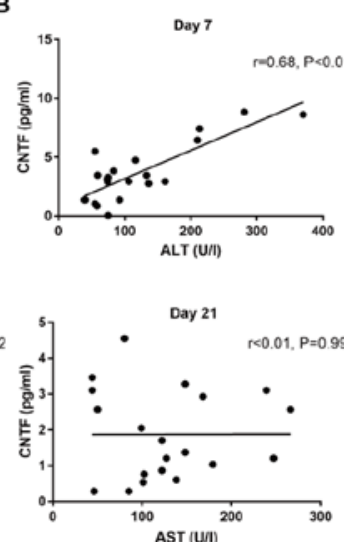
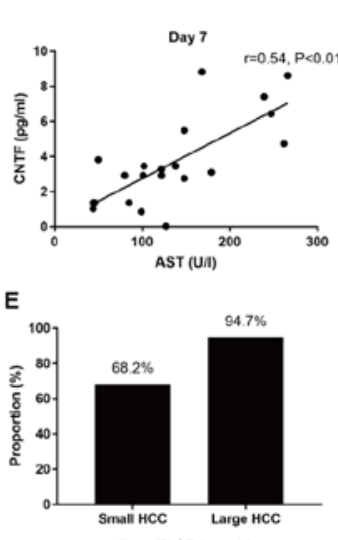

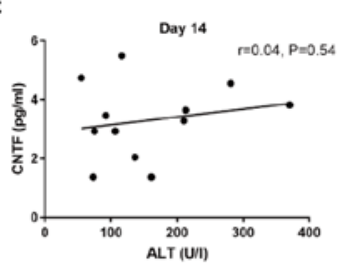

$\mathbf{F}$

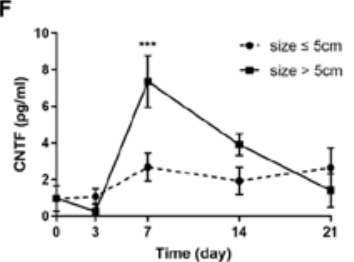

Figure 5. Correlations between the aminotransferase and CNTF serum levels. Scatter plot showing a Spearman's correlation analysis between CNTF and ALT, and AST on day (A) 3, (B) 7, (C) 14 and (D) 21 after TACE. (E) Comparison of severe post-TACE hepatitis between patients with large and small tumors. (F) Patients with large tumors showed increased CNTF levels on day 7 after TACE treatment, whereas those with small tumors showed no significant changes in CNTF levels after TACE. ${ }^{* * *} \mathrm{P}<0.001$. CNTF, ciliary neurotrophic factor; TACE, transarterial chemoembolization; ALT, alanine aminotransferase; AST, aspartate aminotransferase.
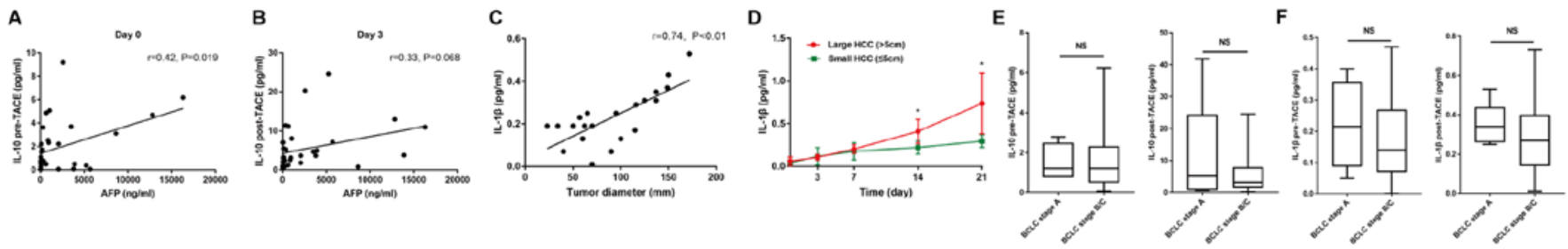

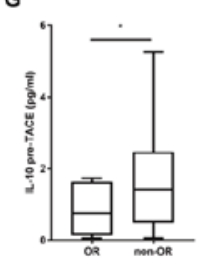

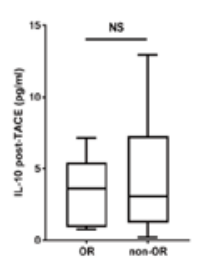

H

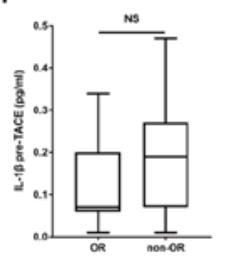

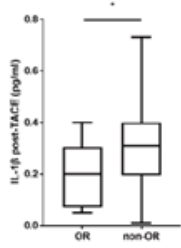

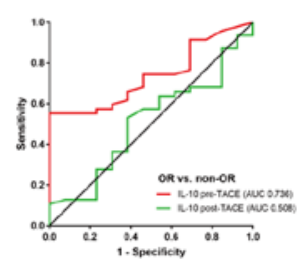

$J$

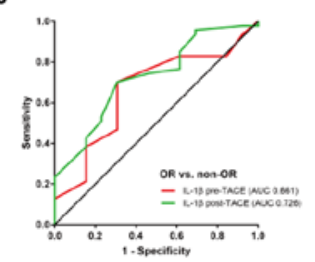

Figure 6. Preinterventional serum levels of IL-10 and post-interventional serum levels of IL-1 $\beta$ predict the objective response after TACE. Spearman's correlation analysis between (A) IL-10 levels and pre-TACE AFP levels, (B) IL-10 levels and AFP levels on day 3 after TACE, and (C) IL-1 $\beta$ levels and tumor diameter. (D) Patients with large HCC tumors showed a more prominent early-phase increase in IL-1 $\beta$ levels after therapy compared with those with small tumors. Serum levels of (E) IL-10 and (F) IL-1 $\beta$ pre-and post-TACE treatment were unaltered between patients with BCLC stage A and B/C. Serum levels of (G) IL-10 and (H) IL-1 $\beta$ pre- and post-TACE treatment in patients with and without OR. ROC curve analysis for (I) IL-10 and (J) IL-1 $\beta$ pre- and post-TACE treatment in patients with and without OR. OR patients, $\mathrm{n}=13$; and non-OR patients, $\mathrm{n}=47$. ${ }^{*} \mathrm{P}<0.05$; ns, P $>0.05$. BCLC, Barcelona Clinic Liver Cancer; OR, objective response; ROC, receiver operating characteristic; AUC, area under the curve, TACE, transarterial chemoembolization; AFP, $\alpha$-fetoprotein; NS, non-significant; HCC, hepatocellular carcinoma; IL, interleukin.

cytokine expression profiles of 9 patients with $\mathrm{HCC}$ at different time points after TACE using a cytokine mRNA microarray were analysed. Subsequently, the cytokine mRNA expression profiles post-TACE on days 3, 7, 14 and 21 were compared to the profiles obtained preintervention (day 0). Venn 2.1 was used for differential gene integration. The data demonstrated that five genes, including NEUROD1, TREM1, NRG3, CLC and TNFAIP6, were present in four groups: Day 3 vs. 0, day 7 vs. 0 , day 14 vs. 0 and day 21 vs. 0 . Borromeo et al (32) reported that NEUROD1 plays crucial roles in promoting malignant behaviour and survival in patients with SCLC. TREM2, a novel pattern recognition receptor family member, is generally regarded to be an enhancer of immune responses (33). According to pharmacogenomics, NRG3 rs1649942 genetic variants have been validated to affect epithelial ovarian cancer first-line treatment outcomes, which confirmed patients carrying the NRG3 rs1649942 A allele presented a significantly longer OS time (34). In addition, CLC and TNFAIP6, which are cytokine receptors, are involved in disease progression and the immune response (35) in a variety of tumors, such as gastric cancer (36), breast cancer (37) and urothelial carcinomas (38).

STEM was used to reveal temporal gene expression profiles and identify significant patterns from the day before TACE (day $0)$ to day 21 post-TACE that were $>2$-fold different $(\mathrm{P}<0.05)$. A total of 6 separate and significant time-varied expression patterns among 507 genes were identified, including profiles 16, 18, 28, 41, 42 and 43. DECs were identified, and their functions were predicted through GO and signaling pathway analyses.

The clustered profile 18 showed initial downregulation before TACE and subsequent upregulation after TACE (Fig. 1). These results might suggest that genes that are downregulated 
A

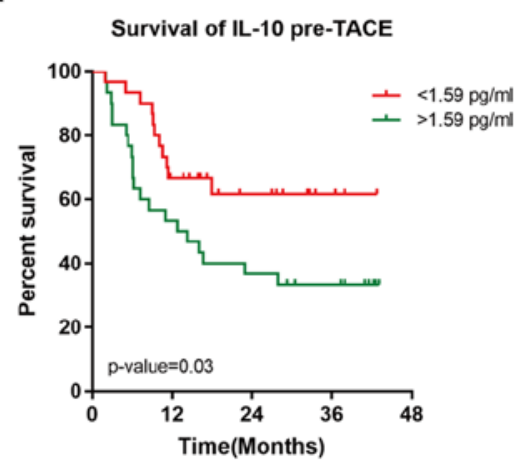

B

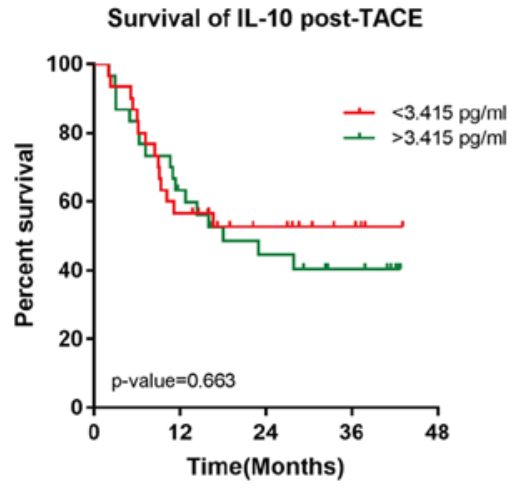

C

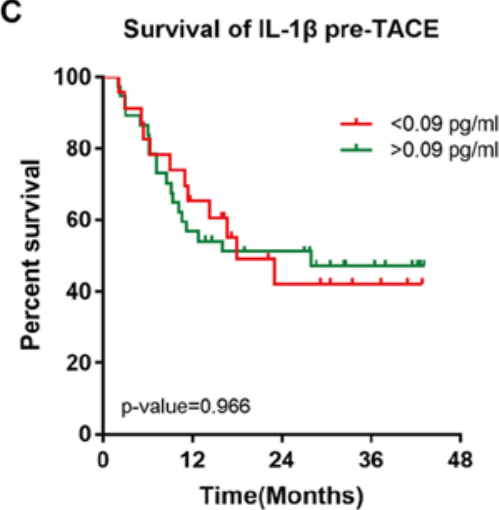

D

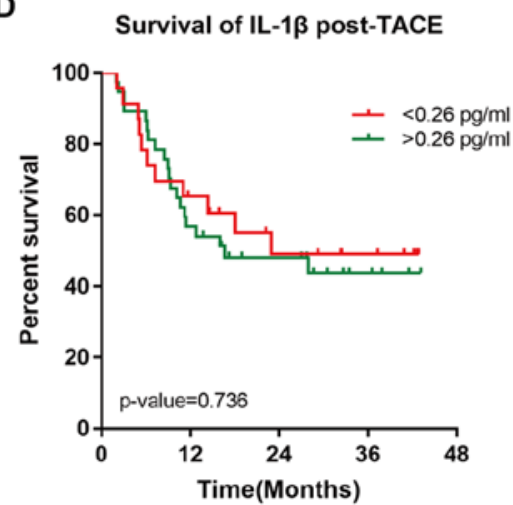

E

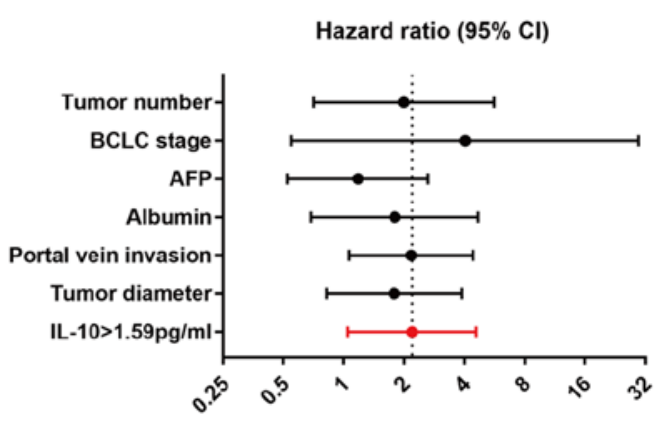

Figure 7. High preinterventional serum levels of IL-10 are associated with an unfavourable prognosis after TACE. Predicting long-term survival using serum levels of IL-10 (A) pre- and (B) post-TACE treatment, divided by ideal cut-off value. Predicting long-term survival using serum levels of IL-1 $\beta$ (C) pre- and (D) post-TACE treatment, divided by ideal cut-off value. (E) Univariate Cox regression analysis revealed that the pre-TACE serum level of IL-10 is a prognostic factor (red color). Black circles indicate the hazard ratio while error bars indicate 95\% CI. TACE, transarterial chemoembolization; IL, interleukin; CI, confidence intervals; BCLC, Barcelona Clinic Liver Cancer; AFP, $\alpha$-fetoprotein.

during the initial phase after TACE might be turned off and could therefore be responsible for hindering the HCC-like phenotype and hepatic injury after a tumor-intervening event. The CNTF, colony stimulating factor 2 receptor $\alpha$ subunit, interferon- $\gamma$, IL-5, PDGFRB, PRL and IL-27 receptor subunit $\alpha$ genes were differentially expressed and enriched in the JAK-STAT signaling pathway in profile 18 (Table SIII). Previous studies showed that JAK-STAT signaling was closely associated with cytokine signalling, as it regulates essential cellular mechanisms, such as proliferation, invasion, survival, inflammation and immunity $(39,40)$. In addition, CNTF is an important hepatoprotective agent in carbon tetra chloride $\left(\mathrm{CCl}_{4}\right)$-induced hepatic injury (41). In a retrospective cohort study, IL-5, PDGFRB and PRL predicted survival benefits in patients with advanced HCC treated with sorafenib (42). Similar to profile 18, the DECs and pathways clustered in profile 16 also included inflammatory signaling pathways (the JAK-STAT and NF-kB pathways), as well as CCR1, CCR7, ICAM1 and BMP4 (Table SIII). CCR1 and CCR7 play important roles in head and neck squamous cell carcinoma progression and regional lymph node metastasis and recurrence. CCR1 were highly expressed in primary carcinomas and CCR7 was associated with disease-free survival time (43). The trend curves of the gene signatures clustered in profiles 28, 41, 42 and 41 were similar and were all gradually upregulated (Fig. 1B-G). The DECs clustered in profile 28, including CXCL1, IL-4, IL-13 and IL-17RA, and in profile 43, including LCN2, CCL2, CXCL2, MMP3 and MMP9, were enriched in the IL-17 signaling pathway (Table SIII). A previous study demonstrated a relationship between the IL-17/IL-17R axis and the tumor inflammatory microenvironment in patients with NSCLC. Immunoreactivity for IL-17A, IL-17F, IL-17RA and IL- 17RC was significantly elevated, while IL-17E was reduced (44). In profile 41 , IL-1 $\beta$, IL-10 and CCL5 were differentially expressed and enriched in the NF-kB, TNF and Toll-like receptor signaling pathways. Meanwhile, JAK-STAT and IL-17 signalling pathways were not associated in profile 41. In line with previous results, IL-1 $\beta$, IL-10 and CCL5 were involved in multiple signaling pathways that affect the development and progression of tumors. IL-1 $\beta$ caused expansion of the bone metastatic niche and led to tumor proliferation in breast cancer (45). IL-10 and integrin pathways have been reported to be strongly associated with head and neck cancer progression (46). In addition, it has been reported that $\mathrm{CC} 15 / \mathrm{CCR} 1 / \beta$-catenin/Slug via mesenchymal stem cells promotes colorectal cancer development (47). In addition to these common signaling pathways, HIF-1 signaling was also significantly enriched in profile 42 . It was previously demonstrated that HIF-1, a key transcription factor involved in hypoxia and inflammation, induces HCC cells to undergo EMT $(48,49)$.

To better understand the role of cytokine expression during TACE, ELISA was performed and demonstrated that the temporal variations in cytokines were similar to the results obtained by STEM. The CNTF gene, which clustered in profile 18, was acutely increased after TACE but decreased after 
Table III. Univariate Cox regression analysis to predict overall survival.

\begin{tabular}{lcc}
\hline \multirow{2}{*}{ Parameter } & \multicolumn{2}{c}{ Univariate Cox regression } \\
\cline { 2 - 3 } & P-value & Hazard ratio (95\% CI) \\
\hline Serum IL-10 $>1.59 \mathrm{pg} / \mathrm{ml}$ & 0.037 & $2.193(1.048-4.589)$ \\
Tumor diameter $(\mathrm{cm})$ & 0.144 & $1.785(0.821-3.882)$ \\
Portal vein invasion & 0.033 & $2.171(1.064-4.431)$ \\
BCLC stage & 0.171 & $4.031(0.548-29.636)$ \\
Albumin $(\mathrm{g} / \mathrm{l})$ & 0.230 & $1.799(0.690-4.691)$ \\
AFP $(\mathrm{ng} / \mathrm{ml})$ & 0.690 & $1.178(0.526-2.636)$
\end{tabular}

AFP, $\alpha$-fetoprotein; BCLC, Barcelona Clinic Liver Cancer; CI, confidence interval.

Table IV. Multivariate Cox regression analysis to predict overall survival.

\begin{tabular}{lcc}
\hline & \multicolumn{2}{c}{ Multivariate Cox regression } \\
\cline { 2 - 3 } Parameter & P-value & Hazard ratio (95\% CI) \\
\hline Serum IL-10 $>1.59 \mathrm{pg} / \mathrm{ml}$ & 0.049 & $2.249(1.005-5.031)$ \\
Tumor diameter $(\mathrm{cm})$ & 0.855 & $0.918(0.369-2.288)$ \\
Portal vein invasion & 0.115 & $1.870(0.858-4.074)$ \\
BCLC stage & 0.229 & $3.682(0.441-30.762)$ \\
Albumin $(\mathrm{g} / \mathrm{l})$ & 0.680 & $1.241(0.445-3.459)$ \\
AFP $(\mathrm{ng} / \mathrm{ml})$ & 0.889 & $1.066(0.435-2.608)$ \\
\hline
\end{tabular}

AFP, $\alpha$-fetoprotein; BCLC, Barcelona Clinic Liver Cancer; CI, confidence interval.

day 7 and reached baseline levels thereafter, while the trend for IL-5 was not statistically significant. Hu et al (50) reported that CNTF regulates PI3K and AMP-activated protein kinase (AMPK) and is therefore involved in metabolic diseases and hepatocarcinogenesis. CNTF acts as a hepatoprotective agent against $\mathrm{CCl}_{4}$-induced hepatic injury, and a correlation between the post-treatment day 7 serum aminotransferase and CNTF levels was observed in the present study. In agreement with previous data (41) the proportion of released CNTF was positively correlated with the degree of hepatic damage, which inhibited lipoprotein secretion and impaired the ability to act as transport vehicles for lipids from the liver to the circulation. According to the trend curve and signaling pathway of profile 41 , the survival and prognostic value of IL-1 $\beta$ and IL-10 was investigated further in clinical features. The results of the present study demonstrated that the pre-TACE serum levels of IL-10 predicted the local tumor response and OS time of a patient, while the post-TACE serum levels of IL- $1 \beta$ only indicated the local tumor response. In addition, multivariate Cox regression analysis revealed that the prognostic value of the pre-TACE IL-10 levels was independent of tumor diameter, BCLC stage, AFP, portal vein invasion and liver function. However, studies of the effect of IL-10 on tumor progression have previously concentrated on antitumor immune effects (51). IL-10 is an immunomodulatory cytokine that has previously been shown to participate in T-cell inactivation and the impairment of adaptive immunity (52-54). Regarding its prognostic value, high IL-10 expression was found to be a marker of longer survival prognosis in breast cancer in addition to decreased tumor cell migration (55). In HCC, IL-10 and IL-12 have been shown to be involved in the progression of chronic inflammation leading to HCC, and they could therefore be considered biomarkers that reflect the degree of inflammation in HCC development (56). Meanwhile, the pre-TACE serum levels of IL-10 exhibited better survival prediction compared with post-TACE levels. One possible reason for this result is that TACE may disturb the tumor microenvironment, which contributed to hypoxia for killing tumor cells. In the current study, the role of the serum IL-10 level as a good prognostic marker seems to be dependent on the tissue microenvironment.

However, there are many limitations to the present study. First, a cytokine microarray was used to detect differential gene expression. In accordance to a previous study, this study also reported that some cytokines were undetectable in a number of patients, and many differences were not significant, such as IL-6 and IL-8 associated with TACE prognosis (19). Second, due to lack of an accepted interventional animal model and limited number of healthy controls, the effect of TACE on cytokine levels in normal liver tissue could not be evaluated. Meanwhile, as TACE is a minimally invasive surgery, it is difficult to obtain a large number of liver tissue samples for further study, such as immunohistochemistry and western blot analyses. Third, the present study was conducted using a small cohort of patients, and only four cytokines were identified using ELISA. Moreover, determining the mechanisms underlying the effects of cytokines in HCC after TACE requires further study.

In summary, a time series of cytokine expression profiles in patients with HCC undergoing TACE was identified. Early phase increases in CNTF serum levels observed after TACE may exert hepatoprotective effects and are associated with post-treatment hepatic injury. Changes in the expression of IL-1 $\beta$ and IL-10 during the process of TACE are associated with long-term outcomes and prognostic survival. These data provide insight into the respective contributions to different biological functions of cytokines released after TACE and their clinical value.

\section{Acknowledgements}

Not applicable.

\section{Funding}

This study was supported by the National Natural Science Foundation of China (grant nos. 81772590 and 81572395), Shanghai Municipality: Shanghai Outstanding Academic Leaders Plan (grant no. 2013-48) and Shanghai Program of Shanghai Academic Research Leader (grant no. 14XD1401100).

\section{Availability of data and materials}

The datasets used and/or analyzed during the present study are available from the corresponding author on reasonable request. 


\section{Author' contributions}

FQ, LW and PH designed the study. FQ, LW, PH and ZZ recruited the patients. FQ performed the experiments. JX, $\mathrm{ZZ}$ and BY designed the stufy and performed the assessment of the radiological TACE response. FQ and $\mathrm{ZZ}$ performed statistical analyses and generated the figures and tables. FQ and JX drafted the manuscript. All authors read and approved the final manuscript.

\section{Ethics approval and consent to participate}

Informed consent was obtained from each patient, and the present study was approved by The Ethics Committees of Zhongshan Hospital.

\section{Patient consent for publication}

Not applicable.

\section{Competing interests}

The authors declare that they have no competing interests.

\section{References}

1. Tang A, Hallouch O, Chernyak V, Kamaya A and Sirlin CB: Epidemiology of hepatocellular carcinoma: Target population for surveillance and diagnosis. Abdom Radiol (NY) 43: 13-25, 2018.

2. Hoyos S, Escobar J, Cardona D, Guzmán C, Mena Á, Osorio G, Pérez C, Restrepo JC and Correa G: Factors associated with recurrence and survival in liver transplant patients with HCC-a single center retrospective study. Ann Hepatol 14: 58-63, 2015.

3. Vitale A, Peck-Radosavljevic M, Giannini EG, Vibert E, Sieghart W, Van Poucke S and Pawlik TM: Personalized treatment of patients with very early hepatocellular carcinoma. J Hepatol 66: 412-423, 2017.

4. Patel T: Increasing incidence and mortality of primary intrahepatic cholangiocarcinoma in the United States. Hepatology 33: $1353-1357,2001$

5. Sacco R, Tapete G, Simonetti N, Sellitri R, Natali V, Melissari S, Cabibbo G, Biscaglia L, Bresci G and Giacomelli L: Transarterial chemoembolization for the treatment of hepatocellular carcinoma: A review. J Hepatocell Carcinoma 4: 105-110, 2007.

6. Han K and Kim JH: Transarterial chemoembolization in hepatocellular carcinoma treatment: Barcelona clinic liver cancer staging system. World J Gastroenterol 21: 10327-10335, 2015.

7. Raoul JL, Forner A, Bolondi L, Cheung TT, Kloeckner R and de Baere T: Updated use of TACE for hepatocellular carcinoma treatment: How and when to use it based on clinical evidence. Cancer Treat Rev 72: 28-36, 2019.

8. Opal SM and DePalo VA: Anti-inflammatory cytokines. Chest 117: 1162-1172, 2000.

9. Ramaswamy P, Goswami K, Dalavaikodihalli Nanjaiah N, Srinivas D and Prasad C: TNF- $\alpha$ mediated MEK-ERK signaling in invasion with putative network involving $N F-\kappa B$ and STAT-6: A new perspective in glioma. Cell Biol Int 43: 1257-1266, 2019.

10. Quail DF, Olson OC, Bhardwaj P, Walsh LA, Akkari L, Quick ML, Chen IC, Wendel N, Ben-Chetrit N, Walker J, et al: Obesity alters the lung myeloid cell landscape to enhance breast cancer metastasis through IL5 and GM-CSF. Nat Cell Biol 19: 974-987, 2017.

11. Shih CM, Lee YL, Chiou HL, Hsu WF, Chen WE, Chou MC and Lin LY: The involvement of genetic polymorphism of IL-10 promoter in non-small cell lung cancer. Lung Cancer 50: 291-297, 2005.

12. Lech-Maranda E, Baseggio L, Bienvenu J, Charlot C, Berger F, Rigal D, Warzocha K, Coiffier B and Salles G: Interleukin-10 gene promoter polymorphisms influence the clinical outcome of diffuse large B-cell lymphoma. Blood 103: 3529-3534, 2004.
13. Cao HY,Zou P and Zhou H: Genetic association of interleukin-10 promoter polymorphisms and susceptibility to diffuse large B-cell lymphoma: A meta-analysis. Gene 519: 288-294, 2013.

14. Matsumoto K, Oki A, Satoh T, Okada S, Minaguchi T, Onuki M, Ochi H, Nakao S, Sakurai M, Abe A, et al: Interleukin-10 -1082 gene polymorphism and susceptibility to cervical cancer among Japanese women. Jpn J Clin Oncol 40: 1113-1116, 2010.

15. Vairaktaris E, Yapijakis C, Serefoglou Z, Derka S, Vassiliou S, Nkenke E, Vylliotis A, Spyridonidou S, Neukam FW, Schlegel KA and Patsouris E: The interleukin-10 (-1082A/G) polymorphism is strongly associated with increased risk for oral squamous cell carcinoma. Anticancer Res 28: 309-314, 2008.

16. Zhang J, Zhang Q, Lou Y, Fu Q, Chen Q, Wei T, Yang J, Tang J, Wang J, Chen Y, et al: Hypoxia-inducible factor-1 $\alpha /$ interleukin-1 $\beta$ signaling enhances hepatoma epithelial-mesenchymal transition through macrophages in a hypoxic-inflammatory microenvironment. Hepatology 67: 1872-1889, 2018.

17. Mohs A, Kuttkat N, Reissing J, Zimmermann HW, Sonntag R, Proudfoot A, Youssef SA, de Bruin A, Cubero FJ and Trautwein C: Functional role of CCL5/RANTES for HCC progression during chronic liver disease. J Hepatol 66: 743-753, 2017.

18. Kim MJ, Jang JW, Oh BS, Kwon JH, Chung KW, Jung HS, Jekarl DW and Lee S: Change in inflammatory cytokine profiles after transarterial chemotherapy in patients with hepatocellular carcinoma. Cytokine 64: 516-522, 2013

19. Loosen SH, Schulze-Hagen M, Leyh C, Benz F, Vucur M, Kuhl C, Trautwein C, Tacke F, Bruners P, Roderburg C and Luedde T: IL-6 and IL-8 serum levels predict tumor response and overall survival after TACE for primary and secondary hepatic malignancies. Int J Mol Sci 19: E1766, 2018.

20. Almeida BR, Barros BCSC, Araújo ACL, Alcantara C and Suzuki E: Paracoccidioides species present distinct fungal adherence to epithelial lung cells and promote different IL-8 secretion levels. Med Microbiol Immunol, 2019.

21. Markov N and Simon HU: IL-37: A new player in the chronic rhinosinusitis arena. J Allergy Clin Immunol: Oct 28, 2019 (Epub ahead of print).

22. Moaaz M, Youssry S, Elfatatry A and El Rahman MA: Th17/Treg cells imbalance and their related cytokines (IL-17, IL-10 and TGF- $\beta$ ) in children with autism spectrum disorder. J Neuroimmunol 337: 577071, 2019.

23. Zhou L, Wang LY, Zhang XM, Zeng NL, Chen TW, Li R, Huang YC and Tang YL: Semi-quantitative assessment of the presence and Child-Pugh class of hepatitis B related cirrhosis by using liver lobe-based dynamic contrast-enhanced MRI. Clin Radiol 71: 1289-1295, 2016.

24. Sato Y, Watanabe H, Sone M, Onaya H, Sakamoto N, Osuga K, Takahashi $\mathbf{M}$ and Arai Y; Japan Interventional Radiology in Oncology Study Group-JIVROSG: Tumor response evaluation criteria for HCC (hepatocellular carcinoma) treated using TACE (transcatheter arterial chemoembolization): RECIST (response evaluation criteria in solid tumors) version 1.1 and mRECIST (modified RECIST): JIVROSG-0602. Ups J Med Sci 118: 16-22, 2013.

25. Jalal H, Pechlivanoglou P, Krijkamp E, Alarid-Escudero F, Enns E and Hunink MG: An overview of $\mathrm{R}$ in health decision sciences. Med Decis Making 37: 735-746, 2017.

26. Ernst J and Bar-Joseph Z: STEM: A tool for the analysis of short time series gene expression data. BMC Bioinformatics 7: 191, 2006.

27. Huang da W, Sherman BT and Lempicki RA: Systematic and integrative analysis of large gene lists using DAVID bioinformatics resources. Nat Protoc 4: 44-57, 2009.

28. Kanehisa M, Furumichi M, Tanabe M, Sato Y and Morishima K: KEGG: New perspectives on genomes, pathways, diseases and drugs. Nucleic Acids Res 45: D353-D361, 2017.

29. von Mering C, Huynen M, Jaeggi D, Schmidt S, Bork P and Snel B: STRING: A database of predicted functional associations between proteins. Nucleic Acids Res 31: 258-261, 2003.

30. Dolcino M, Tinazzi E, Puccetti A and Lunardi C: In systemic sclerosis, a unique long non coding RNA regulates genes and pathways involved in the three main features of the disease (vasculopathy, fibrosis and autoimmunity) and in carcinogenesis. J Clin Med 8, 2019.

31. Silva JP, Berger NG, Tsai S, Christians KK, Clarke CN, Mogal H, White S, Rilling W and Gamblin TC: Transarterial chemoembolization in hepatocellular carcinoma with portal vein tumor thrombosis: A systematic review and meta-analysis. HPB (Oxford) 19: 659-666, 2017. 
32. Borromeo MD, Savage TK, Kollipara RK, He M, Augustyn A Osborne JK, Girard L, Minna JD, Gazdar AF, Cobb MH and Johnson JE: ASCL1 and NEUROD1 reveal heterogeneity in pulmonary neuroendocrine tumors and regulate distinct genetic programs. Cell Rep 16: 1259-1272, 2016.

33. Tang W, Lv B, Yang B, Chen Y, Yuan F, Ma L, Chen S, Zhang S and Xia J: TREM2 acts as a tumor suppressor in hepatocellular carcinoma by targeting the PI3K/Akt/ $\beta$-catenin pathway. Oncogenesis 8: 9, 2019.

34. Pinto R, Assis J, Nogueira A, Pereira C, Coelho S, Brandão M, Dias J, Alves S, Pereira D and Medeiros R: Pharmacogenomics in epithelial ovarian cancer first-line treatment outcome: Validation of GWAS-associated NRG3 rs1649942 and BRE rs7572644 variants in an independent cohort. Pharmacogenomics J 19: 25-32, 2019.

35. Al-Nashmi M, Taha S, Alsharoqi I and Bakhiet M: Interleukin 1 receptor antagonist and 2'-5'-oligoadenylate synthetase-like molecules as novel biomarkers for multiple sclerosis patients in Bahrain. Mult Scler Relat Disord 18: 1-7, 2017.

36. Gu Z, Li Y, Yang X, Yu M, Chen Z, Zhao C, Chen L and Wang L: Overexpression of CLC-3 is regulated by XRCC5 and is a poor prognostic biomarker for gastric cancer. J Hematol Oncol 11: 115 2018 .

37. Yang H, Ma L, Wang Y, Zuo W, Li B, Yang Y, Chen Y, Chen L, Wang L and Zhu L: Activation of ClC-3 chloride channel by $17 \beta$-estradiol relies on the estrogen receptor $\alpha$ expression in breast cancer. J Cell Physiol 233: 1071-1081, 2018.

38. Chan TC, Li CF, Ke HL, Wei YC, Shiue YL, Li CC, Yeh HC, Lee HY, Huang SK, Wu WJ and Li WM: High TNFAIP6 level is associated with poor prognosis of urothelial carcinomas. Urol Oncol 37: 293.e11-293.e24, 2019.

39. Pencik J, Pham HT, Schmoellerl J, Javaheri T, Schlederer M, Culig Z, Merkel O, Moriggl R, Grebien F and Kenner L: JAK-STAT signaling in cancer: From cytokines to non-coding genome. Cytokine 87: 26-36, 2016.

40. Wubetu GY, Utsunomiya T, Ishikawa D, Yamada S, Ikemoto T, Morine Y, Iwahashi S, Saito Y, Arakawa Y, Imura S, et al: High STAT4 expression is a better prognostic indicator in patients with hepatocellular carcinoma after hepatectomy. Ann Surg Oncol 21 (Suppl 4): S721-S728, 2014

41. Cui MX, Jiang JF, Min GN, Han W and Wu YJ: Ciliary neurotrophic factor analogue aggravates $\mathrm{CCl}_{4}$-induced acute hepatic injury in rats. Can J Physiol Pharmacol 95: 620-623, 2017.

42. Hayashi T, Yamashita T, Terashima T, Suda T, Okada H, Asahina Y, Hayashi T, Hara Y, Nio K, Sunagozaka H, et al: Serum cytokine profiles predict survival benefits in patients with advanced hepatocellular carcinoma treated with sorafenib: A retrospective cohort study. BMC Cancer 17: 870, 2017.

43. González-Arriagada WA, Lozano-Burgos C, Zúñiga-Moreta R, González-Díaz P and Coletta RD: Clinicopathological significance of chemokine receptor (CCR1, CCR3, CCR4, CCR5, CCR7 and CXCR4) expression in head and neck squamous cell carcinomas. J Oral Pathol Med 47: 755-763, 2018.

44. Huang Q, Ma XC, Yang X, Wang W, Li Y, Lv Z, Corrigan CJ, Chen $\mathrm{Y}$ and Ying S: Expression of IL-17A, E, and F and their receptors in non-small-cell lung cancer. J Biol Regul Homeost Agents 32: 1105-1116, 2018.
45. Tulotta C, Lefley DV, Freeman K, Gregory WM, Hanby AM, Heath PR, Nutter F, Wilkinson JM, Spicer-Hadlington AR, Liu X, et al: Endogenous production of IL1B by breast cancer cells drives metastasis and colonization of the bone microenvironment. Clin Cancer Res 25: 2769-2782, 2019.

46. Bornstein S, Schmidt M, Choonoo G, Levin T, Gray J, Thomas CR Jr, Wong M and McWeeney S: IL-10 and integrin signaling pathways are associated with head and neck cancer progression. BMC Genomics 17: 38, 2016

47. Chen K, Liu Q, Tsang LL, Ye Q, Chan HC, Sun Y and Jiang X: Human MSCs promotes colorectal cancer epithelial-mesenchymal transition and progression via CCL5/ $\beta$-catenin/Slug pathway. Cell Death Dis 8: e2819, 2017.

48. Zhang Q, Bai X, Chen W, Ma T, Hu Q, Liang C, Xie S, Chen $\mathrm{C}, \mathrm{Hu} \mathrm{L}, \mathrm{Xu} \mathrm{S}$ and Liang T: Wnt/ $\beta$-catenin signaling enhances hypoxia-induced epithelial-mesenchymal transition in hepatocellular carcinoma via crosstalk with hif-1 $\alpha$ signaling. Carcinogenesis 34: 962-973, 2013.

49. Chen W, Ma T, Shen XN, Xia XF, Xu GD, Bai XL and Liang TB: Macrophage-induced tumor angiogenesis is regulated by the TSC2-mTOR pathway. Cancer Res 72: 1363-1372, 2012

50. Hu X, Zhao Y, He X, Li J, Wang T, Zhou W, Wan D, Wang H and $\mathrm{Gu}$ J: Ciliary neurotrophic factor receptor alpha subunit-modulated multiple downstream signaling pathways in hepatic cancer cell lines and their biological implications. Hepatology 47: 1298-1308, 2008.

51. Mumm JB, Emmerich J, Zhang X, Chan I, Wu L, Mauze S, Blaisdell S, Basham B, Dai J, Grein J, et al: IL-10 elicits IFN $\gamma$-dependent tumor immune surveillance. Cancer Cell 20: 781-796, 2011.

52. Wang S, Gao X, Shen G, Wang W, Li J, Zhao J, Wei YQ and Edwards CK: Interleukin-10 deficiency impairs regulatory $\mathrm{T}$ cell-derived neuropilin-1 functions and promotes Th1 and Th17 immunity. Sci Rep 6: 24249, 2016.

53. Huber S, Gagliani N, Esplugues E, O'Connor W Jr, Huber FJ, Chaudhry A, Kamanaka M, Kobayashi Y, Booth CJ, Rudensky AY, et al: Th17 cells express interleukin-10 receptor and are controlled by Foxp3 ${ }^{-}$and Foxp3+ regulatory CD4+ T cells in an interleukin-10-dependent manner. Immunity 34: 554-565, 2011.

54. Dennis KL, Blatner NR, Gounari F and Khazaie K: Current status of interleukin-10 and regulatory T-cells in cancer. Curr Opin Oncol 25: 637-645, 2013.

55. Ahmad N, Ammar A, Storr SJ, Green AR, Rakha E, Ellis IO and Martin SG: IL-6 and IL-10 are associated with good prognosis in early stage invasive breast cancer patients. Cancer Immunol Immunother 67: 537-549, 2018.

56. El-Emshaty HM, Nasif WA and Mohamed IE: Serum cytokine of IL-10 and IL-12 in chronic liver disease: The immune and inflammatory response. Dis Markers 2015: 707254, 2015.

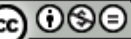

This work is licensed under a Creative Commons

Attribution-NonCommercial-NoDerivatives 4.0 International (CC BY-NC-ND 4.0) License. 